\title{
Case report: intramedullary nailing of subtrochanteric fracture in lateral traction
}

\begin{abstract}
Subtrochanteric femur fracture fixation remains one of the most challenging procedures to orthopedic surgeons. Apart of the choices of implant used, patient positioning for intramedullary nailing of subtrochanteric femoral fracture plays a crucial role in fracture reduction and should be considered during preoperative planning. It is partly contributed to the deforming muscle forces acting one the proximal fragment of femur. As compared to supine position, intramedullary fixation in lateral decubitus with traction of affected leg is believed achieve to easier reduction of proximal femur fragments due to the deforming muscle forces, easier attainment of intramedullary fixation entry point due to les interference of the thorax and chest wall especially in obese patient. We report the case of a 28 -year-old male patient who underwent intramedullary fixation of right subtrochanteric fracture in lateral decubitus with traction of the affected leg.
\end{abstract}

Keywords: Subtrochanteric femur fracture, lateral decubitus, traction
Volume 10 Issue 6 - 2018

\author{
Chungkia NG \\ University of Malaya, Malaysia
}

\begin{abstract}
Correspondence: Chungkia NG, University of Malaya, Malaysiajalan Universiti 59100 Kuala Lumpur,Wilayah Persekutuan Kuala Lumpur, Malaysia, Email chungkea85@hotmail.com
\end{abstract}

Received: February 01, 2018| Published: November 12, 2018

\section{Introduction}

Subtrochanteric fracture accounts for $25 \%$ of proximal femur fracture and their distribution is bimodal which involves young made adults and old females predominantly. ${ }^{1}$ Subtrochanteric fracture is unstable as result of the high compressive and tensile forces of muscle that separate the fracture segments. Therefore surgery is the optimal option for the patients with no absolute contraindication. Intramedullary fixation has been gaining popularity for the past decades due to its unique advantage- short force arm which can better distribute the stress compared to extramedullary fixation. ${ }^{2}$ Intramedullary fixation in supine traction position has been well described. However the literature describing intramedullary fixation in lateral decubitus position with traction is scarce. ${ }^{3}$ This is partly contributed to its drawback as the positioning set up is more time consuming. Otherwise performing intramedullary fixation in lateral decubitus position with traction table provides an alternative to orthopedic surgeons for easier access to entry point and fracture reduction. We reported a 28 -yearold man who underwent intramedullary fixation for subtrochanteric fracture in lateral decubitus position in traction table.

\section{Case report}

A 28-year-old man had injured his right lower limb after a motor vehicle accident. Post trauma, he complained of pain over right proximal thigh and was unable to lift up his right leg. Physical examination revealed a deformity over right thigh with intact neurovascular function. Standard pelvis and right femur radiographs show a fracture over right subtrochanteric femur (Figure 1). Otherwise lesser trochanter and piriformis fossa were intact.

The patient underwent intramedullary fixation under general anesthesia using cephalomedullary nail. He was positioned in lateral position on traction table, with the operative leg over the top of the perineal post (Figure 2). Boot was attached to the table with traction applied; hip was slightly adducted and moderately flexed. The leg was then internally rotated 10-15 degrees. Contra-lateral knee was flexed with boot attached. Padding was placed over fibular neck prominence over non operative leg. Closed reduction was achieved under $\mathrm{C}$-arm fluoroscopy guidance. C-arm was brought in perpendicular to the long axis of the femur. When moving proximally and distally, the entire $\mathrm{C}$-arm was moved, to stay perpendicular to the long axis. To visualize the proximal femur and the head, $\mathrm{C}$-arm was rotated $15^{\circ}$ over the top and tilted $45^{\circ}$ cephalad. After prepping and draping, $3-5 \mathrm{~cm}$ of skin incision was made with greater trochanter as the center. Entry point was identified following by guide wire insertion. The force line of the limbs and fracture position were properly maintained followed by intramedullary reaming and intramedullary nail insertion. Afterwards the proximal and distal locking screws were inserted and fracture position was confirmed under C-arm fluoroscopy.

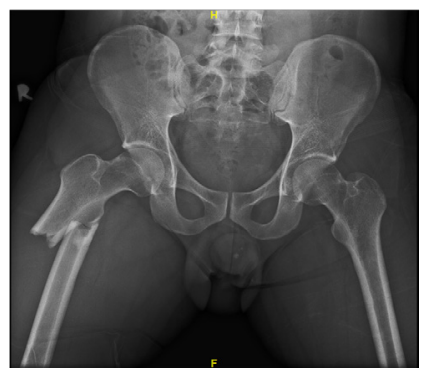

Figure I Pelvis radiograph showed subtrochanteric fracture of right femur. Proximal fragment was abduction and externally rotated.

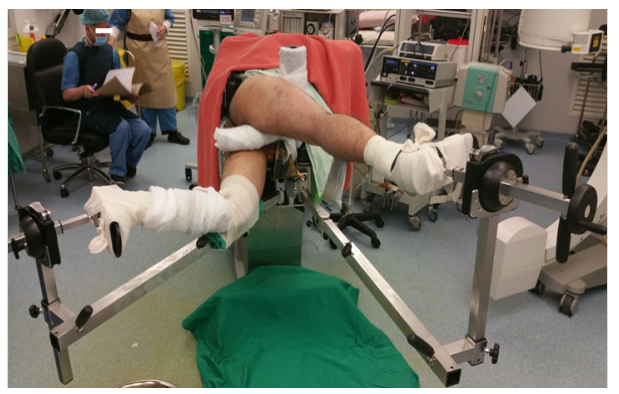

Figure 2 Patient was positioned in lateral decubitus with traction of affected leg. Operative leg was positioned on perineal post, abducted and moderately flexed. Contra lateral leg was flexed without traction. 
Postoperatively patient was given intravenous prophylactic antibiotics for 3 doses. He was initiated on the exercise of muscle strength of lower limbs and functional exercises of hip joint immediately after operation. He was discharged uneventfully post operative day 2. Weight bearing over operative leg was prohibited 8-12 weeks.

\section{Discussion}

Intramedullary fixation of subtrochanteric fracture remains one of the most challenging procedures to orthopedic surgeons. It is partly due to the several deforming forces subjected to the proximal femur fragments: flexion (provoked by the iliopsoas), abduction (by the gluteus medius), and external rotation (by the external rotators). The adductors, inserted in the distal region of the femur, are responsible for the varus deformity. ${ }^{4}$ Delayed union or non union has been reported as a common complication of subtrochanteric fracture as a result of limited contact surface area, decreased vascularity, and high mechanical stresses which might impair the bone healing. ${ }^{5}$ Therefore proper reduction prior to fixation is detrimental to correct the rotation and flexion of proximal femur fragments and hence to achieve satisfactory bone consolidation.

Several surgical techniques especially patient's position during intramedullary fixation have been described in literatures to ease the reduction of subtrochanteric fracture. Patient may be placed in either supine or lateral position with or without traction table. Previous literature described placing patient in lateral decubitus position on traction table; operative hip was flexed, adducted and knee was flexed with traction applied to take account of any flexion of the proximal fracture fragment. Instead of applying traction over flexed knee, we applied traction over the operative leg with knee extended.

Positioning patient in lateral decubitus with traction of the operative leg offers several advantages to orthopedic surgeons. Firstly it improves access to the entry point especially in obese patient as the trunk is shifted away from surgical site which minimizes the interference of entry point by the torso. Second there is a good control of proximal fragment with less interference from the flank, chest wall or ribs. Thirdly, placing patient in lateral decubitus with traction of affected leg could neutralize the proximal femur fragment which tends to angulate in varus in supine position. However fixation of subtrochanteric fracture in lateral position is without its disadvantages.
The techniques might compromise the pulmonary function. Secondly venous congestion could be caused from the perineal post compressing the medial thigh and femoral vessels. Apart from that sciatic nerve over the operated leg might be at risk due to prolonged traction and hip flexion. Finally many believe positioning in lateral decubitus is more time consuming. However we believe that once familiarity is gained the set-up time might not be different from the supine position.

\section{Conclusion}

The key point to reduce the complication of subtrochanteric fracture fixation is the quality of the reduction. Deforming muscle forces make treatment of subtrochanteric fractures challenging. Therefore patient positioning is crucial to achieve satisfactory reduction. Intramedullary fixation in lateral decubitus with traction of the affected leg offers an alternative to orthopedic surgeons for easier attainment of reduction and intramedullary nail entry point.

\section{Acknowledgements}

None.

\section{Conflict of interest}

Author declares that there is no conflict of interest.

\section{References}

1. JW Fielding. Subtrochanteric Fractures. Clin Orthop Relat Res. 1973;86-99.

2. Wang J, Ma XL, Ma JX, et al. Biomechanical Analysis of Four Types of Internal Fixation in Subtrochanteric Fracture Models. Orthop Surg. 2014;6(2):128-136.

3. A Alex Jahangir, Edward A P, Thomas A R. Intramedullary Nailing of Subtrochanteric Fractures: Relevant Anatomy and Entry Portals, Supine, or Lateral Positioning. Techniques in Orthopaedics. 2008;23:113-17.

4. Joglekar SB, Lindvall EM, Martirosian A. Contemporary Management of Subtrochanteric Fractures. Orthop Clin North Am . 2015;46(1):2135.

5. Bedi, T Toan Le. Subtrochanteric Femur Fractures. Orthop Clin North Am. 2044;35:473-483. 